\title{
Mulheres e cirurgiãs
}

\section{Women and surgeons}

Talta Franco, ECBC-RJ'; Elizabeth Gomes dos Santos, TCBC-RJ²

\author{
RE S U M O
}

As autoras apresentam o panorama da inserção das mulheres nas Especialidades Cirúrgicas desde o final do século XIX até os dias atuais e analisam as dificuldades enfrentadas bem como o aumentodesta inserção nos dias atuais.

Descritores: Cirurgia. Mulheres Cirurgiãs.

\section{INTRODUÇÃO}

A Cirurgia moderna surgiu mais ou menos na mesma época em que as mulheres conquistaram o direito de freqüentar as universidades, no final do século XIX. Naquele tempo, nem os cirurgiões nem as mulheres eram muito prestigiados. Os primeiros, porque exerciam uma atividade manual considerada pouco nobre, por lidar com o corpo humano e seus humores, com a dor e com a morte. As mulheres eram vistas como pessoas de segunda classe, comparadas às crianças e aos silvícolas, não geriam o próprio dinheiro, não podiam fazer curso superior nem ingressar no serviço público. No Brasil só adquiriram direito ao voto em 1932.

A Cirurgia progrediu rapidamente a partir do aprimoramento da anestesia, do controle da infecção e da reposição sangüínea. Seu grande impulso foi a $1^{\text {a }}$ Grande Guerra, quando a Medicina já tinha recursos para manter vivos os feridos que, antes, não teriam sobrevivido. O progresso tecnológico fez o resto.

Até o início do século XIX, quando o exercício da Medicina ainda não dependia de licenças dadas por cursos oficiais, que não aceitavam mulheres, há várias referências a mulheres cirurgiãs que trabalhavam no anonimato ou escudadas atrás de parentes masculinos. Em algumas épocas e lugares eram, inclusive, ameaçadas de morte se fossem descobertas. A atração pela cirurgia portanto, sempre existiu. O caso mais notório e curioso é o de Miranda Barry (1797-1865) que, sob o nome de James Barry, foi o principal Oficial médico e Cirurgião da Armada Britânica onde serviu durante 40 anos mostrando "grande habilidade cirúrgica, maneiras agressivas e pontaria perfeita". Só após sua morte descobriram que era mulher e que, possivelmente, teria tido pelo menos uma gestação. Tal constatação causou grande impacto mas o escândalo foi abafado e ela foi enterrada como homem ${ }^{1}$.
Quando o ensino formal foi instituído, as dificuldades aumentaram até que o ingresso nas universidades Ihes fosse autorizado, nas décadas de 1850, nos Estados Unidos, e 1870, na Inglaterra, na França e no Brasil. A campanha pelo direito à Universidade foi apenas o primeiro passo porquanto mais alguns anos e muitas lutas se passaram até que o direito ao internato em hospitais, imprescindível para a formação de cirurgiões, Ihes fosse concedido. Mesmo assim há registros anteriores, como os de Mary Edward Walker (1832-1919), cirurgiã do exército americano na guerra civil; Emeline Horton Cleveland (1829-1878) que realizava grandes cirurgias abdominais no Women's Hospital of Philadelphia; Marie Josepha Mergler (18511901), catedrática de cirurgia ginecológica e, depois, decana da Northwsetern University Woman's Medical College, em Chicago; Elsie Inglis (1864-1917), cirurgiã da $1^{\text {a }}$ Grande Guerra e fundadora do Scottish Women's Hospital² e, provavelmente, várias outras que esta pesquisa não localizou.

Para as mulheres médicas, o caminho foi lento, com idas e vindas, fases de abertura e outras de retração, falta de apoio familiar e estatal, preconceitos variados.

Entre 1879, quando a lei Leôncio de Carvalho permitiu-lhes o ingresso nas faculdades, e 1900, cinco muIheres completaram o ensino médico e exerceram a profissão: Rita Lobato Lopes, gaúcha de Rio Grande, em 1887; Ermelinda Lopes de Vasconcelos, gaúcha de Porto Alegre, em 1888; Antonieta Cesar Dias, outra gaúcha de Rio Grande, em 1889; Maria Amélia Cavalcante, pernambucana de Serinhaem, em 1889; Judith Adelaide Maurity Santos, fluminense de Cantagalo, em 1900.

Antes, Maria Augusta Generoso Estrela, carioca, e Josefa Águeda Felisbela Mercedes de Oliveira, de Recife, precisaram sair do país para se formarem nos Estados Unidos, respectivamente em 1881 e 1882.

Entre 1900 e 1938, 76 mulheres se formaram na Faculdade de Medicina da UFRJ, com a média de duas por

\footnotetext{
1. Professora Titular de Cirurgia Plástica da Universidade Federal do Rio de Janeiro - UFRJ- Rio de Janeiro - RJ- BR. 2. Cirurgiã do Hospital Universitário Clementino Fraga Filho da Universidade Federal do Rio de Janeiro - UFRJ- Rio de Janeiro - RJ- BR; Mestre em Cirurgia Geral pela Faculdade de Medicina da UFRJ.
} 
ano. Entre 1949 e 1968, as mulheres representavam, em média, 10\% de cada turma. Em 1969, começou a curva crescente que, em dois anos, aumentou de 16,48\% para $38,53 \%$ e se estabilizou, entre 1972 e 1981, em torno dos 40\%. Entre 1982 e 1993, esse percentual já chegava aos $50 \%$, aproximando-se dos 60\% a partir de 1994, e alcançando 63,51\% em 1999 (Figura 1) Em 2007, confirmou-se o discreto predomínio de mulheres $(51 \%)^{3}$.

Entretanto, o aumento percentual de cirurgiãs não acompanhou o crescimento acelerado do número de médicas formadas nas três últimas décadas. Dados do Colégio Brasileiro de Cirurgiões (CBC) revelam que, em outubro de 2008, eram membros 5513 homens e 657 mulheres (11,90\%).

CBC foi fundado em 30/07/1929 e as primeiras mulheres a se associarem ao Colégio foram:

Merisa Braga De Miguez Garrido - Cirurgia Vascular - 23/11/1959-RJ; Oldea Bertolazzo - Obstetrícia29/6/1964 - RJ; Maria Luiza Pessoa Cavalcanti Cancerologia -30/7/1966 - RJ; Talita Romero Franco Cirurgia Plástica -1/1/1968 - RJ; Angelita Habr-Gama Coloproctologia - Em 15/7/1969 -SP.

Os dados do CBC são mais fidedignos em relação à Cirurgia Geral posto que não existe uma outra entidade de classe específica para ela. Nas outras especialidades, as ditas Sociedades Brasileiras agrupam a maioria dos especialistas em atividade, os quais nem sempre são filiados ao CBC, sobretudo se forem de outros estados. Como exemplo, a Sociedade Brasileira de Cirurgia Plástica registra em seu quadro 916 mulheres, enquanto no CBC apenas 29 estão registradas.

A partir da década de 1950 o CREMERJ passou a registar as especialidades dos inscritos. O número de muIheres em especialidades cirúrgicas, muito baixo nas déca- das de 1950 e 1960 (três em cada década), subiu exponencialmente na década de 1970, coincidindo com o aumento do número de mulheres cursando as faculdades de Medicina, que atingiram 50\% do alunado. O número de registros cresceu de 79, em 1970, para 112, em 1980, e 164, na década de 1980. Curiosamente, a década de 2000 apresentou diminuição em $50 \%$, baixando para 87 registros (Figuras 2 e 3).

Em publicação recente, Jesus 4 relata a diminuição efetiva do contingente de cirurgiões gerais, homens e mulheres, em vários países do mundo. Explicações são muitas, como o aumento de interesse por especialidades menos exigentes em tempo e menos sujeitas a estresse e problemas médico-legais. Além disto, o número de mulheres médicas vem se mantendo maior em relação ao de homens e elas, tradicionalmente, não mostram preferência pela área cirúrgica. Neumayer ${ }^{5}$ et al. relataram que, embora o contingente feminino nas escolas de medicina nos Estados Unidos seja de quase $50 \%$, somente 15\% delas escolhem alguma especialidade cirúrgica.

Explicar porque poucas mulheres desejam ser cirurgiãs é um tema complexo que não tem resposta úni$c a^{6}$. Para Neumayer, a falta de cirurgiãs que sejam modelos a serem seguidos é um importante fator limitante à escolha da Cirurgia como carreira pelas estudantes de medicina ${ }^{3}$. Park ${ }^{7}$ et al. analisando questionário respondido por "staffs" do sexo feminino que já exerciam a Cirurgia Geral e por estudantes de Medicina do último ano, concluiu que o interesse feminino pela cirurgia é igual ao masculino. O que detém as mulheres na escolha pela $\mathrm{Ci}$ rurgia Geral é a procura de uma especialidade que lhes permita conciliar uma atividade profissional gratificante com a vida pessoal, aí incluídos casamento e maternidade ${ }^{4}$. Os dados obtidos por Ferris ${ }^{8}$ et al. em estudo semelhante ao

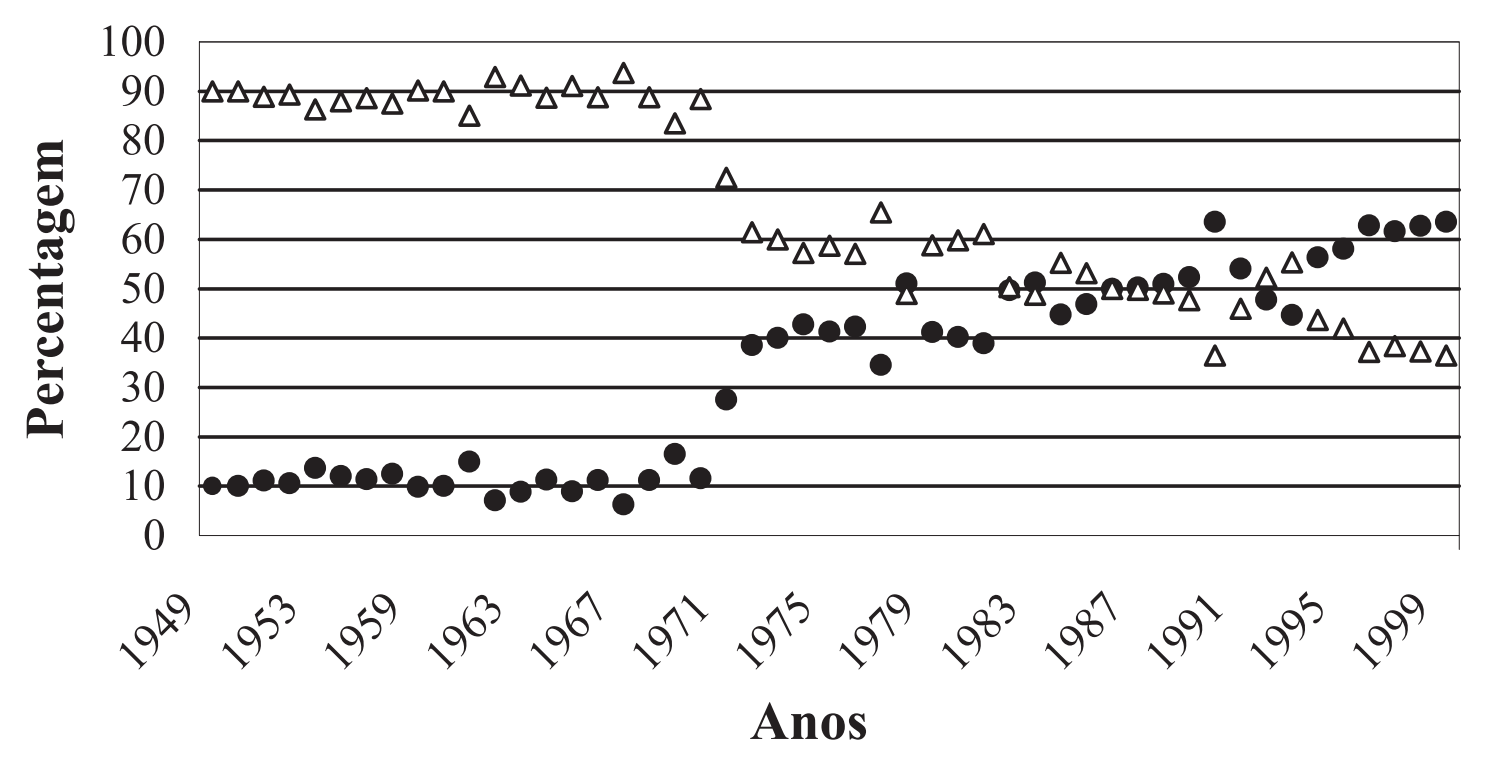

$\multimap$ Feminino Masculino

Figura 1 - Relação percentual alunos/alunas. 


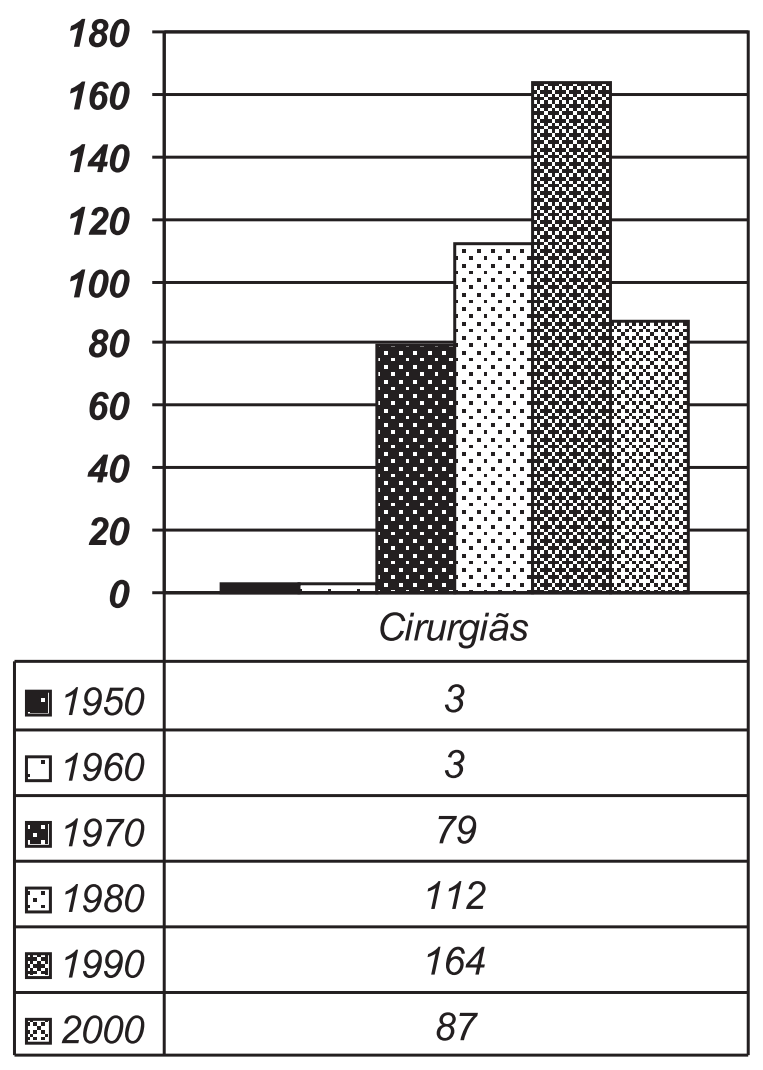

Figura 2 - Inscrição CREMERJ. Mulheres Cirurgiãs.

de Parker mostram que 15\% das entrevistadas (413) sentiram-se discriminadas por ocasião da admissão para residência mas que quase a metade (199) afirmou que suas carreiras não foram prejudicadas por qualquer tipo de discriminação. No mesmo estudo 82\% (338/413) das entrevistadas afirmou ser necessária a presença de um modelo feminino.

Diferentemente dos homens que, simplesmente, escolhem uma profissão ou especialidade que lhes agrade ou convenha, as mulheres mesmo quando mais capazes têm inúmeros obstáculos a vencer. Embora nos dias de hoje o preconceito de gênero tenha sido quase extinto, no Ocidente, a educação das mulheres, em algumas famílias, ainda favorece a baixa auto-estima, a insegurança intelectual, a dependência emocional e financeira. Na sociedade, seus atributos mais valorizados são físicos, em detrimento de destreza, habilidade manual, inteligência e todos os outros necessários para o sucesso como cirurgiã. Durante séculos, mesmo no Ocidente, as mulheres conviveram com direitos civis limitados e quase nenhuma credibilidade profissional. Chantagens, assédios, difamação e ridicularizações fizeram parte da evolução das mulheres na Medicina, particularmente na Cirurgia, de forma mais grave no início, mas ainda presentes, dissimuladas em algumas brincadeiras.

"As mulheres não podem, seriamente, seguir a carreira médica, a não ser que deixem de ser mulheres. Devido às leis fisiológicas, mulheres

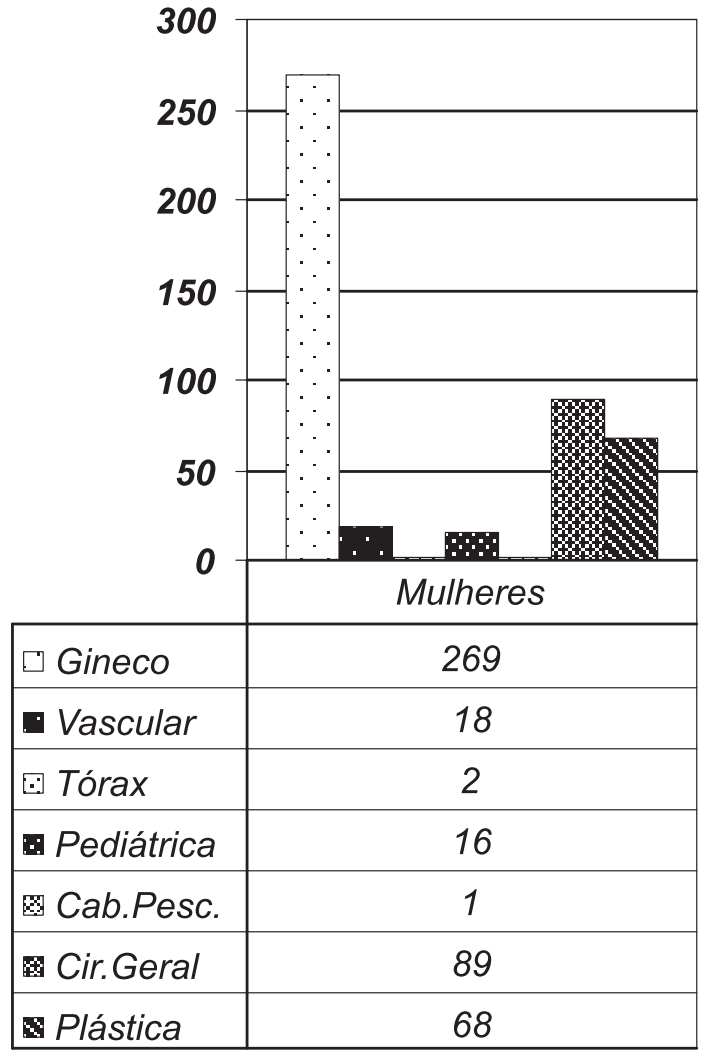

Figura 3 - Mulheres e Especialidades Cirúrgicas (CREMERJ - 2008).

médicas são ambíguas, hermafroditas ou assexuadas, monstros sob todos os pontos de vista"

Idéias e sentimentos deste tipo fazem parte de um passado remoto, mas, de algum modo, ainda subexistem. Certas conquistas profissionais e culturais femininas continuam a provocar reação de surpresa. Ao contrário, a ausência de mulheres em posições de representatividade social, não causa nenhum espanto. $\mathrm{Na}$ UFRJ em 1998, cento e noventa anos após sua fundação, só quatro mulheres chegaram a Professoras Titulares por concurso, e apenas uma delas é cirurgiã.

Segundo Pringle ${ }^{10}$, o fenótipo masculino inspira $25 \%$ a mais de confiança do que o feminino. Isto significa que, para qualquer cargo que pleiteie, uma mulher precisa mostrar ser pelo menos, 25\% mais capacitada do que seu concorrente masculino mais próximo, para ter as mesmas chances de sucesso. Aspectos pessoais considerados favoráveis para os cirurgiões, tais como personalidade forte, auto-controle, mente questionadora, capacidade de liderança e uma certa agressividade são vistos como qualidades nos homens e como componentes estranhos à personalidade feminina, gerando muitas vezes dúvidas quanto à sua feminilidade.

Até a década de 1960, as poucas cirurgiãs existentes encontravam ambiente hostil. Não havia vestiário feminino, roupas adequadas ou quaisquer outras facilidades. Eram sempre confundidas com enfermeiras ou 
instrumentadoras. Nunca se pensava nelas, em princípio, como cirurgiãs e também eram alvos de comentários desagradáveis. Os pacientes freqüentemente diziam preferir operar com homens. Não havia cirurgiãs bem sucedidas que servissem como exemplo e estímulo

A tabela do CREMERJ, (figura 4) analisando a idade das cirurgiãs registradas, espelha o que se vê nos números relacionadas à década de registro. Há um pico de cirurgiãs na faixa entre 30 e 40 anos de idade, decrescendo até quase anular-se na faixa acima dos 70 anos, decréscimo explicado, tanto devido a um percentual inevitável de óbitos, como pelo menor número de cirurgiãs registradas nas décadas de 1950 e 1960. Causa preocupação, entretanto, que o número de jovens cirurgiãs, esteja diminuindo significativamente, agora quando os preconceitos contra as mulheres em especialidades cirúrgicas parecem estar acabando.

A distribuição de participantes nas diferentes sociedades cirúrgicas brasileiras em outubro de 2008 , pode ser vista na tabela 1.

A Cirurgia Pediátrica se mostra um campo de boa inserção feminina. Dos 85 participantes da Associação de Cirurgia Pediátrica do Rio de Janeiro, 43 são do sexo feminino, configurando o maior percentual de profissionais do sexo feminino em especialidade cirúrgica.

Entende-se o bom percentual de mulheres em Cirurgia Plástica, especialidade vista no imaginário popular, sobretudo por sua vertente estética, pela maioria de pacientes femininos e por ser apresentada erroneamente como uma cirurgia de menor "gravidade".

A Coloproctologia exercida por mãos femininas talvez seja menos constrangedora para as pacientes e parece ter tido impulso a partir do momento em que a Professoara Angelita Gama tornou-se nacionalmente conhecida. Os exemplos bem sucedidos são fator importante de incentivo.

A Ortopedia, apesar de sua aparelhagem cada vez mais delicada e sofisticada, ainda é vista como dependente de força física, o que talvez afaste as possíveis candidatas, embora o número de mulheres ortopedistas tenha aumentado nos últimos anos.

A Cirurgia Geral, nos últimos anos, apresentou crescimento no número de cirurgiãs. Este aumento, entretanto, não pode ser considerado verdadeiro já que a Cirurgia Geral é pré-requisito obrigatório para todas as especialidades cirúrgicas e, com a ultrasetorização, o perfil da especialidade está cada vez menos nítido o que dificulta avaliar o significado deste crescimento.

Existem ainda algumas especialidades cujo acesso feminino continua a ser raro. A Cirurgia Torácica, a Cirurgia Cardíaca e a Neurocirurgia têm um contingente muito pequeno de especialistas do sexo feminino isto ocorre também em outras profissões, como aviadoras, engenheiras de mega-construções, altas executivas de empresas etc. (Tabela 2).

Em uma análise simples dos Serviços de Cirurgia Geral dos Hospitais Universitários constatamos que no Hospital Pedro Ernesto da UERJ são ao todo 20 cirurgiões, sendo três mulheres; no HUCFF-UFRJ entre os 30 cirurgiões há

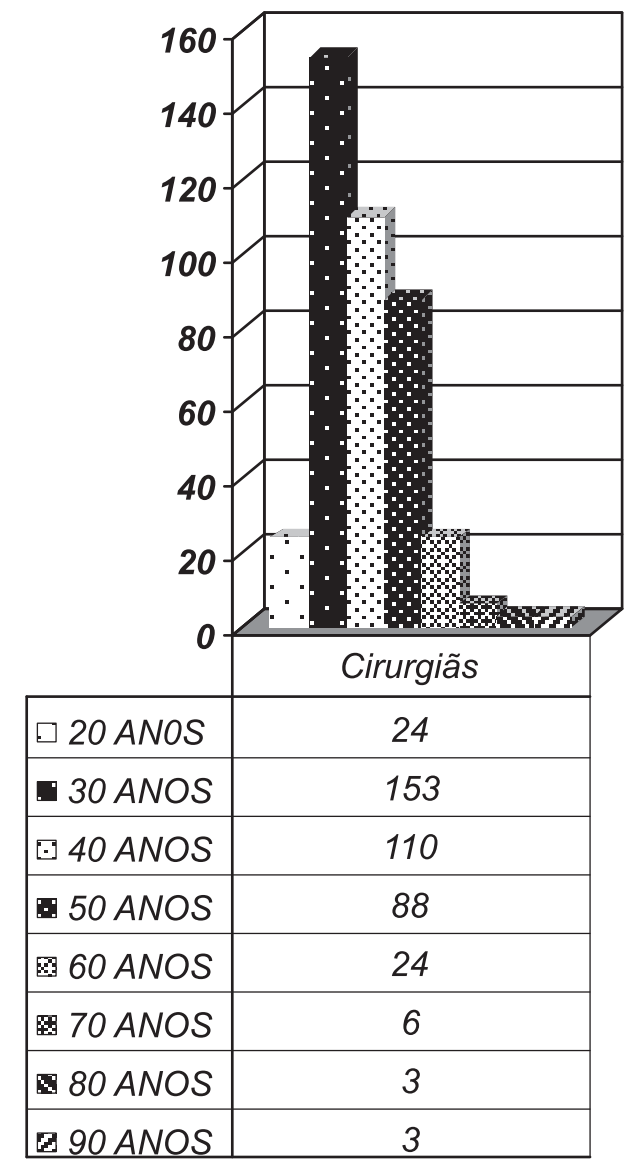

Figura 4 - $\quad$ Registro de Cirurgiãs por idade (CREMERJ,2008).

apenas uma mulher, no Gafrée Guinle da UniRio o total de cirurgiões é 13 e não há nenhuma mulher; o mesmo ocorre no Hospital Antonio Pedro UFF onde são 24 cirurgiões entre os quais não há mulheres.

Existe também uma grande competição interpares. Acresce o fato de que a relação cirurgião-paciente é de um para um. Enquanto vários clínicos podem examinar o mesmo paciente, apenas um cirurgião pode operá-lo. Marcar território sempre foi característica masculina e poucas mulheres se interessam por este tipo de combate e quando o fazem correm sempre o risco de serem rotuladas erradamente.

Tabela 1 - Distribuição de participantes nas diferentes sociedades cirúrgicas brasileiras.

\begin{tabular}{lcc}
\hline & Homens & Mulheres \\
\hline Pediátrica & $49,42 \%$ & $50,58 \%$ \\
Plástica & $73,93 \%$ & $26,07 \%$ \\
Coloproctologia & $74,10 \%$ & $25,80 \%$ \\
Cirurgia Geral (CBC) & $87,48 \%$ & $12,52 \%$ \\
Cirurgia Torácica & $92,93 \%$ & $7,07 \%$ \\
Neurocirurgia & $94,59 \%$ & $5,41 \%$ \\
Ortopedia & $96,03 \%$ & $3,97 \%$ \\
Cirurgia Cardiovascular & 96,16 & $3,84 \%{ }^{11}$ \\
\hline
\end{tabular}


A simultaneidade do crescimento profissional com a formação de uma família afeta muito mais as mulheres do que os homens tendo em vista o conceito ainda arraigado de que criar filhos e cuidar da casa são atividades prioritariamente femininas. Isto leva as muIheres a postergarem a gravidez e, às vezes, até a inviabilizá-la. Lerner, Stolzmann e Gulla ${ }^{12}$ analisaram 365 mulheres urologistas quanto ao número de filhos, evolução da gravidez e do parto, reprodução assistida e complicações. Concluíram que, em relação à população em geral, as mulheres urologistas tiveram filhos mais tarde, em menor número, alto índice de reprodução assistida e incidência maior de complicações. Atribuem estes fatos à faixa etária mais alta das gestantes, devido às necessi- dades profissionais e sugerem que os programas de residência médica dêem maior suporte às mulheres que desejem engravidar nesta fase que, biologicamente, é a mais propícia ${ }^{13}$.

As dificuldades a serem vencidas pelas mulheres que desejam ser cirurgiãs, uma certa falta de auto-confiança feminina e de modelos estimulantes em cirurgiãs de sucesso, além dos problemas advindos da falta usual de suporte institucional às mães médicas, são fatores ainda presentes, contribuintes para o pequeno número de muIheres cirurgiãs. Estes fatores de impedimento sendo progressivamente derrubados, a escolha da Cirurgia como especialidade pelas mulheres dependerá, então, apenas de seus interesses pessoais.

Tabela 2 - Distribuição de Cirurgiãs por Especialidade no Brasil. Fonte: CBC.

\begin{tabular}{|c|c|c|c|c|c|c|c|c|c|c|c|c|c|}
\hline & Geral & Vasc & Ped & Plast & Colo & Ginec & Obst & Mama & Torax & $C A$ & Card & Ortop & Total \\
\hline$A C$ & & 1 & 1 & & & & & & & & & & 2 \\
\hline $\mathrm{AL}$ & 6 & 3 & & & 1 & & & & & & & & 10 \\
\hline AM & 9 & 1 & 1 & & & 1 & & & & & & & 12 \\
\hline BA & 8 & & 1 & 1 & 3 & & & 1 & & & & & 14 \\
\hline$C E$ & 11 & & & & & & & & & & & & 11 \\
\hline DF & 10 & 1 & 1 & & 1 & 1 & & & & 1 & & & 15 \\
\hline ES & 13 & & & & & & & & & & & & 13 \\
\hline GO & 5 & & & & & & & & & & & & 5 \\
\hline MA & 1 & 1 & & & & & & & & & & & 2 \\
\hline$M G$ & 27 & & & & & 2 & & & & & & & 29 \\
\hline MS & 12 & & & & 1 & & & & & & & & 13 \\
\hline MT & 1 & & 1 & & & & & & & & & & 2 \\
\hline $\mathrm{OU}$ & 2 & & & & & & & & & 1 & & & 3 \\
\hline PA & 7 & & & 1 & & & & & & & 1 & & 9 \\
\hline PB & & & & & 1 & & & & & & & & 1 \\
\hline PE & 2 & 2 & & & 1 & & 1 & & & & & & 6 \\
\hline $\mathrm{PI}$ & 1 & & & & 1 & 1 & & & & & & & 3 \\
\hline PR & 24 & & 1 & & 1 & & & & & 1 & & & 27 \\
\hline RJ & 186 & 7 & 8 & 17 & 8 & 7 & 3 & 2 & 3 & 10 & & 1 & 252 \\
\hline RN & 6 & & 1 & & & & & & & & & & 7 \\
\hline RO & 1 & 1 & & & & & & & & & & & 2 \\
\hline $\mathrm{RR}$ & & & 1 & 1 & & & & & & & & & 2 \\
\hline RS & 14 & & 1 & 1 & & 1 & & & & & & & 17 \\
\hline SC & 4 & & & & & & & & & & & & 4 \\
\hline SE & 6 & & & & 1 & & & & & & & & 7 \\
\hline SP & 151 & 3 & 2 & 8 & 8 & 3 & & & & 3 & & & 178 \\
\hline TO & 1 & & & & & & & & & & & & 1 \\
\hline Tota & 508 & 20 & 19 & 29 & 27 & 16 & 4 & 3 & 3 & 16 & 1 & 1 & 647 \\
\hline
\end{tabular}

\section{A $B$ B S T}

The authors present the current view of the women in Surgery since the end of the XIX century until now. They discuss the difficulties they face when they choose surgery as a career and the progress now achieved.

Key words: Surgery, Women Surgeons. 


\section{REFERENCIAS}

1. Garza H. Women in medicine. New York: Franklin Watts; 1994

2. Stille DR. Extraordinary women of medicine. New York: Grolier Publishing; 1997.

3. Franco T. Médicas pioneiras. In: Gomes MM, Vargas SSM, Valladares AF. A faculdade de medicina primaz do Rio de Janeiro. Rio de Janeiro: Atheneu; 2001. p. 37-61.

4. Jesus LE. Ensinar Cirurgia: como e para quem? Rev Col Bras Cir. 2008; 35(1):136-40.

5. Neumayer L, Kaiser S, Anderson K, Barney L, Curet M, Jacobs D et al. Perceptions of women medical students and their influence on career choice. Am J Surg. 2002; 183(2):146-50.

6. Marschall JG, Karimuddin AA. Decline in popularity of general surgery as a career choice in North America: review of postgraduate residency training selection in Canada, 1996-2001. World J Surg. 2003; 27(3): 249-52. Epub 2003 Feb 27.

7. Park J, Minor S, Taylor RA, Vikis E, Poenaru D. Why are women deterred from general surgery training? Am J Surg. 2005; 190(1):141-6.

8. Ferris LE, Mackinnon SE, Mizgala CL, McNeill I. Do Canadian female surgeons feel discriminated against as women? CMAJ. 1996; 154(1):21-7

9. Lucas-Championnaire, Just, "article 9997". J Méd Chir Prat. June 1875 issue. p. 241-2.

10. Pringle R. Sex and medicine. Cambridge: University Press; 1998

11. $C B C$ - Colégio Brasileiro de Cirurgiões. Disponível em: http:// www.cbc.org.br - Acesso em 10 de março de 2009.

12. SBCP - Sociedade Brasileira de Cirurgia Plástica. Disponível em: http://www. cirurgiaplastica.org.br - Acesso em 11 de março de 2009.
13. SBCP - Sociedade Brasileira de Coloproctologia. Disponível em: http://www.sbcp.org.br - Acesso em 12 de março de 2009.

14. SBCT - Sociedade Brasileira de Cirurgia Torácica. Disponível em: http://www.sbct.org.br - Acesso em 13 de março de 2009.

15. CIPE - Associação Brasileira de Cirurgia Pediátrica. Disponível em: http://www.cipe.org.br - Acesso em 19 de maio de 2009.

16. SBN - Sociedade Brasileira de Neurocirurgia. Disponível em: http:/ /www.sbn neurocirurgia.com.br - Acesso em 14 de março de 2009.

17. SBCV - Sociedade Brasileira de Angiologia e Cirurgia Vascular. Disponível em: http://www.sbacv.com.br - Acesso em 15 de março de 2009.

18. SBOT - Sociedade Brasileira de Ortopedia e Traumatologia. Disponível em: http://www.sbot.org.br - Acesso em 14 de março de 2009.

19. MEC - Ministério de Educação. Disponível em: http:// www.mec.gov.br - Acesso em 05/01/2009.

20. Lerner LB, Stolzmann KL, Gulla VD. Birth trends and pregnancy complications among women urologists. J Am Coll Surg. 2009; 208(2):293-7. Epub 2008 Dec 4.

Recebido em 25/05/2009

Aceito para correspondência em 30/06/2009

Conflito de interesse: nenhum

Fonte de financiamento: nenhuma

\section{Como citar esse artigo:}

Franco T, Santos EG. Mulheres e cirurgiãs. Rev Col Bras Cir. [periódico na Internet] 2010; 37(1). Disponível em URL: http://www.scielo.br/rcbc

\section{Endereço para correspondência:}

Dra. Talita Franco

E-mail: talita@openlink.com.br 